\title{
Fluid inclusion induced hardening in pyrite: Results from atom probe tomography
}

\author{
RENELLE DUBOSQ ${ }^{1}$, ANNA ROGOWITZ ${ }^{2}$, DAVID
} SCHNEIDER $^{1}$, KEVIN SCHWEINAR $^{3}$ AND BAPTISTE GAULT $^{3,4}$

${ }^{1}$ University of Ottawa

${ }^{2}$ University of Vienna

${ }^{3}$ Max-Planck-Institut für Eisenforschung $\mathrm{GmbH}$

${ }^{4}$ Royal School of Mines, Imperial College London

Presenting Author: renelle.dubosq@gmail.com

The feedbacks between nanoscale deformation and diffusion processes can have a strong influence on the bulk rheology of geomaterials. For instance, strain hardening can occur when dislocations are arrested by the strain field of immobile defects or by the accumulation of mobile solutes in their cores. Interactions with fluids can also affect the mechanical properties of minerals, typically facilitating ductile deformation through nanoscale mechanisms such as hydrolytic weakening, dissolution creep, etc. Until recently, direct observation of these processes was challenging due to limitations in the spatial resolution of conventional structural and geochemical techniques. However, recent developments in microscopy and microanalysis now allow for the compositional measurements and spatial imaging of materials at the near-atomic scale. Herein, we investigate the role of fluid inclusions on mineral rheology in a naturally deformed polycrystalline pyrite aggregate from the Abitibi Subprovince in Canada. We have used a correlative approach combining electron backscatter diffraction (EBSD) mapping, electron channeling contrast imaging (ECCI), scanning transmission electron microscopy (STEM) and atom probe tomography (APT). The combined microstructural data reveal a dominantly brittle regime with minor strain accommodation via crystal-plasticity where dislocations are mostly emitted by the propagating fracture, which is evidenced by minor crystal misorientation and lowangle grain boundary development in the vicinity and at the tip of microfractures. These interpretations are consistent with the peak temperature conditions of the sample estimated at $30227^{\circ} \mathrm{C}$, which falls within the lower range of the brittle to crystal-plastic behaviour of pyrite $\left(260-450^{\circ} \mathrm{C}\right)$. The combined STEM and APT data reveal ubiquitous nanoscale fluid inclusions enriched in As, $\mathrm{O}, \mathrm{Na}$ and $\mathrm{K}$ that are linked to As-enriched dislocations. Based on these data, we propose a fluid inclusion induced hardening model whereby migrating dislocations are pinned by the strain field of fluid inclusions during crystal-plastic deformation, initiating pipe diffusion of trace elements from the fluid inclusions into dislocations leading to their stabilization and resulting in local hardening. Although additional experiments are required on other mineral phases, our initial efforts advance the understanding of the interplay between nanostructures and impurities and its impact on the rheology of geomaterials during relatively low temperature deformation. 